\title{
Factors Affecting Journal Submission Numbers: Impact Factor and Peer Review Reputation
}

\author{
Thomas E. Gaston \\ Francesca Ounsworth \\ Tessa Senders \\ Sarah Ritchie \\ Emma Jones
}

Correspondence to:

Thomas Gaston, John Wiley \& Sons Ltd., 9600 Garsington Road, Oxford, OX4 2DQ.

Abstract

Background: Previous research has found that researchers rank journal reputation and Impact Factor amongst the key selection criteria when choosing where to submit. We explored the actual effect upon submission numbers of several possible factors.

Methods: We retrieved ten years of submission data from over a thousand journals, as well as data on Impact Factor, retractions, and other factors. We performed statistical analysis and identified correlations. We also undertook case study research on the fifty-five most significant submission decreases.

Results: We found a statistically significant correlation between changes in Impact Factor, ISI percentage ranking, and changes in submissions numbers in subsequent years. We also found a statistically significant effect on submission numbers in the year following the publication of a retraction. Our case studies identified other factors, including negative feedback on the peer review process.

Discussion: Our findings regarding Impact Factor confirm previous indications about the significance of Impact Factor on submissions. We explain the correlation with retractions through the concept of "peer review reputation". These results indicate that editors and publishers need to focus on a journal's peer review practices, as well as a journal's Impact Factor, if they are to maintain and grow submissions.

\section{Key Points}

Increased Impact Factor correlates to increased submissions; decreased Impact Factor correlates to decreased submissions

Negative peer review reputation correlates to a decrease in submissions

Editors and publishers need to invest in peer review to maintain submission numbers

This is the author manuscript accepted for publication and has undergone full peer review but has not been through the copyediting, typesetting, pagination and proofreading process, which may lead to differences between this version and the Version of Record. Please cite this article as doi: 10.1002/leap.1285 


\section{Introduction}

The number of submissions received by a journal, while not always directly related to the quality of said manuscripts, often affects the overall number of articles published. The number of manuscripts submitted is therefore an important metric in the sustainability of a journal. Whether it is a subscription journal that wishes to maintain (or increase) its frequency of publication, or an open access journal that wishes to increase the number of articles it can publish (without jeopardizing academic rigor), the success of a journal, both in terms of publication output and in terms of revenue, is ultimately dependent on the number of submissions it receives. For this reason, knowing the factors affecting submission numbers will be of significance both to journal editors and publishers.

Previous research has attempted to identify the factors considered by authors when choosing where to submit, primarily through surveys. A number of surveys conducted by Swan and Brown (Swan, 1999), (Swan \& Brown, 2004) found that the two most important factors affecting the decision of where to submit were readership and journal quality; the question of readership was focused on reaching the right readers rather than the overall number. Solomon and Bjork (Solomon \& Björk, 2011) found the top three factors were fit to subject area of the journal, quality of the journal (sometimes measured by Impact Factor), and speed of review. Respondents to a survey by Søreide \& Winter (Søreide \& Winter, 2010) ranked journal reputation, followed by Impact Factor, as the two most important factors. However, after grouping several factors, the authors found that journal "prestige", followed by turnaround time, were the two most important factors. Factors considered least important were acceptance rate, option to suggest reviewers, and open access. Özçakar et al (Özçakar, Franchignoni, Kara, \& Muñoz, 2012) found that the three most important factors were mission and contents of the journal, Impact Factor, and match between perceived quality of the study and journal Impact Factor. Ziobrowski and Gibler (Gibler \& Ziobrowski, 2002) surveyed real-estate authors and found that the author perception of journal quality was the highest ranked factor. A survey of Canadian researchers found that journal prestige and Impact Factor greatly outranked other criteria (Phase5Research, 2014).

Unpublished research by Wiley, surveying authors of accepted manuscripts, includes a question as to the reasons for submitting to a journal. The top five ranked reasons are shown below, as of May 2019, along with percentage of respondents selecting that option; respondents could select multiple options.

Scope of Journal $(70 \%)$

Reputation of journal (64\%)

Impact Factor (54\%)

Previous experience with journal (36\%)

Expected speed (29\%)

Focusing specifically on open access journals, (Schroter \& Tite, 2006) used interview and survey techniques to study author perceptions regarding where to submit. They found the most important factors were Impact Factor, reputation, readership, speed of publication, and the quality of the peer review system. Regarding willingness to pay APCs, they found that journal quality was the most important factor. A survey conducted by the SOAP project found that $30 \%$ of respondents don't submit to OA journals due to the lack of high-quality OA journals in their field (Vogel, 2011).

Not many of the surveys separated peer review from other factors, such as journal reputation or journal quality. (Nicholas et al., 2015) found that peer review was second only to relevance to the field, when choosing a journal. Being published by a traditional publisher and being highly cited were third and fourth. These respondents linked peer review with high quality. Focusing on OA journals, whether the journal was peer 
reviewed was the key selection criteria - even more important than whether the journal had a reputable publisher. Other research demonstrates that being peer reviewed is the key criteria authors use for distinguishing legitimate and predatory journals (Edie \& Conklin, 2019).

An alternative methodology to surveying researchers was to try and model optimum submission strategies. (Salinas \& Munch, 2015) modelled around maximising expected number of citations, whilst minimising number of required revisions and time in review (also see (Heintzelman \& Nocetti, 2009)). (Coupe, 2004) argued that if authors behaved rationally, they would rank the risk of rejection more highly, because of the implications for delay in getting their manuscript published.

The absolute significance of Impact Factor, distinct from other factors, is difficult to access. A survey of ecologists by (Aarssen et al., 2008) found that most respondents ranked Impact Factor as "very important" or "important". (Calcagno et al., 2012) found that high impact journals publish more resubmissions from other journals (with similar IF); low impact journals publish more "first-intent" submissions. They hypothesized plausibly that this result was due to high impact journals competing for the same submissions. It is well known that Impact Factor is used by universities and other institutions to evaluate performance (Adam, 2002); (Smith, 2006), and therefore publishing in a high IF journal can have implications for promotion and tenure. (Gibler \& Ziobrowski, 2002) found that some author groups prioritise promotion and tenure considerations, whereas others prioritise ease and fairness of the process. (Pepermans \& Rousseau, 2015) found a difference between authors of "high quality" papers, who are looking for a journal with a high impact and/or high standing, and authors of "standard quality" papers, for whom acceptance rate is of equal standing to Impact Factor. Impact Factor is also a key criterion used when creating lists of journal rankings, which are often used to determine where authors submit - though the use of such lists has been criticised for its negative impact on the literature (Willmott, 2011). One exception is the survey of librarians by Neville and Crampsie (Crampsie, 2019). When respondents were allowed to select multiple options, Impact Factor ranked 15th (18\%), behind journal scope (1st; 95\%), whether the journal is peer-reviewed (2nd; 87\%), and intended audience (3rd; 75\%). When respondents were restricted to one, "most important", option, Impact Factor ranked 5th (4\%), behind scope (1st; 49\%), whether the journal is peer-reviewed (2nd; 21\%), and publisher reputation (3rd; 8\%).

It is notable that reputation and/or quality is often ranked higher than Impact Factor by respondents. Whilst Impact Factor can be one factor in establishing journal reputation, if it were the primary or only factor in a journal's reputation, one would expect these two reasons to be ranked on a par. The implication is that there are other aspects of a journal, perhaps not so easily quantified, that contribute to author perceptions of journal reputation, and thus to perceptions of suitability for submission. These might include the prestige of the editorial board, the reception of the journal on social media, and the negative impact of bad press.

Whilst surveying author perceptions is useful, what respondents say they will do in abstract situations does not necessarily indicate what they will do in actual situations. For instance, an author might value turnaround times but nevertheless submit to the highest IF journal in their field. In this study we wanted to explore actual submission numbers and correlate them with likely factors. Based on previous research, we identified three categories to explore and from those categories identified nine factors we wanted to test:

1. Impact Factor

. Absolute Impact Factor

ii. ISI \% subject category ranking

2. Journal Reputation

iii. Net Promoter Score (NPS)

iv. Average reputation score

v. Retractions

vi. Altmetric Score 


\section{Editorial Process}

vii. Time from submission to first decision
viii. Time from submission to acceptance
ix. Acceptance rate

Hypothesis vi (Altmetric score) was later dropped due to availability of data.

\section{Methods}

We retrieved annual submission data for all Wiley journals using ScholarOne Manuscripts between 2007 and 2018 ( $n=1004$ journals). The percentage increase was calculated for each journal for each year between 2008 and 2018 (e.g. the value for 2008 was the difference between submissions in 2007 and 2008.) Some journals were not publishing, or were not on ScholarOne Manuscripts, for all the years between 2007 and 2018, thus null values were marked "n/a" and discounted from the calculation for that particular year. Values for the first and second year of publication were also discounted to avoid skewing. A manual inspection of the data was conducted to remove outliers. We also retrieved data on the median days from submission to first decision, the median days from submission to acceptance, and the acceptance ratio.

We retrieved all the retractions from Wiley journals listed on the Retraction Watch database. We also retrieved retractions listed under "Wiley-Blackwell" and "Blackwell Publishing", and then de-duplicated the results.

We retrieved the Impact Factor and ISI \% primary subject category ranking for 2008 to 2018 for each of the journals in our sample. The ISI \% ranking is a measure created by Clarivate which uses the Impact Factor of journals, within the subject categories defined by Web of Science, to rank them from lowest to highest (where $100 \%=1^{\text {st }}$ journal in subject ranking). We used the percent ranking for comparison as it allows for more nuance than the actual category rank. Not all journals have an Impact Factor/ISI ranking or had an Impact Factor/ISI ranking for all years in our sample range.

We retrieved the average reputation score and the Net Promoter Score from Wiley's internal survey of accepted authors. This data is only available for 2016, 2017, and 2018.

An initial analysis was conducted by categorizing each annual change in submissions into either an increase or a decrease, then comparing this against each of the factors under investigation. Based upon this investigation, we proceeded with a statistical analysis of Impact Factor, ISI \% ranking, and retractions.

For Impact Factor, the number of submissions in the two years after the Impact Factor being published were analyzed (e.g. 2010 and 2011, following IF 2009), resulting in 17 different pairs (e.g. IF 2009 and submissions in 2010). For each of the pairs of years, simple linear regression was performed regressing the number of submissions a journal received in a given year on the Impact Factor of the journal for a given year. The Impact Factor is released midway through the subsequent year (e.g. IF 2017 was released midway through 2018), which is why we analyzed the two subsequent years of submissions. One journal was removed from this part of the analysis as it is an outlier; its 2017 IF was over 200.

The same method was followed to analyse the effect of ISI \% Ranking on the number of submissions. Any journals with a reported ranking of 0 were removed from the data under the assumption that this reflected unranked journals, rather than journals lowest in their category.

For retractions, the percent increase in submissions (2008-2018) was compared with the number of retractions published (2007-2017). Some outliers were removed. The impact of a journal having retractions on the percent increase in the number of submissions received the year after the journal had retractions was analyzed, resulting in 11 different pairs (e.g. 2007 retractions and 2008 submissions). Over the course of the 11 years, only 280 of the total 1004 journals analyzed had retractions. In fact, there are so few journals in a given year. that have 
retractions that a simple linear regression does not provide an accurate analysis of the impact of retractions on the number of submissions a journal receives. For each year, the continuous data regarding the number of retractions in a journal was changed into binary categorical data so that each journal was labeled as either having retractions in that given year ("Yes") or having no retractions in that given year ("No"). For each of the pairs of years, a Welch two sample t-test (a variation on the traditional t-test) was performed in RStudio (R Core Team, 2017) comparing the average percent increase in the number of submissions a journal receives if it had issued retractions and the average percent increase in the number of submissions a journal received if it had issued no retractions. This test was used to demonstrate any statistically significant difference in the means of the two groups, journals with retractions and journals with no retractions. Based on manual inspection of the data, we assumed that retractions are independent events, and that number of retractions in any given year does not affect retractions in subsequent years.

To further our investigation, we proceeded to conduct case studies. We limited ourselves to cases where the number of submissions declined by $10 \%$ or more from a level of 1000 or more submissions. This resulted in 55 cases across 38 journals. We emailed the publishing manager for each journal, including any data described above that we thought might be relevant, and asked for their analysis as to why submissions declined in the given year. We received responses for 39 out of 55 cases, which were evaluated and categorized. We also undertook some desk research to identify any widely-publicised reasons that might explain the decrease in the given year.

\section{Results}

Impact Factor: Absolute Impact Factor

Each simple linear regression being performed by regressing the number of submissions a journal receives (ranging from 2010-2018) on Impact Factors (ranging from 2009-2017) were statistically significant at a 0.05 significance level. Table 1 shows the coefficients found for each simple linear regression. For every 1-point increase in a journal's Impact Factor, the number of submissions the journal receives increases by the value in the table on average (range: $97.4-$ 119.2; mean 105.4).

\section{Impact Factor: ISI subject category ranking}

Linear regressions were then performed with ISI ranking and number of submissions, using the same date ranges as for Impact Factor. For each one percent increase in ISI ranking, Table 2 shows the average increase in number of submissions received for each given year (range: $4.06-5.40$; mean 4.68). All linear regressions were significant to $(p)<0.001$.

\section{Journal Reputation: Net Promoter Score (NPS)}

We were given guidance that twenty responses or more were required to be significant, which meant that there were only 601 cases (that is, $<14 \%$ ) where there was significant data in a given year across the journals included in our sample.

The available data around average reputation score and Net Promoter Score (NPS) was too slight to make any meaningful analysis (most journals would have been excluded due to lack of sufficient respondents.) Of 601 available NPS scores, 591 were "promoter" scores. Of the 10 "demoter" scores, only 3 corresponded with years when the number of submissions decreased at the same journal.

\section{Journal Reputation: Average Reputation Score}

The reputation score was between 1-5. However, there is little variation in our sample. Most average scores were between 4.6 and 4.8 , with no correlation between average reputation score and submission numbers. 
Journal Reputation: Retractions

In total we found 937 retractions, though these included retractions for non-journal publications. After standardizing the journal titles, we identified the retractions, by year of publication, with the journals in our sample. There were 661 retractions across 280 journals between 2007 and 2018 for the our sample, with some journals having multiple retractions in the same year. (We did not attempt an analysis of whether multiple retractions have a bigger affect as the number of cases with multiple retractions would have been too small to be significant.)

Each Welch two sample t-test was performed for each pair of years as described in the Methods section. Table 3 shows the mean percent increase in the number of submissions a journal received if it had one or more retractions in the previous year, as well as the mean percent increase in the number of submissions a journal received if it had no retractions. The p-values are reported; 5 out of the 11 pairs are statistically significant. (For clarification: 0.01 would represent a $1 \%$ increase and -0.01 would represent a $1 \%$ decrease; for example.) For every pair of years that is statistically significant, the percent increase in the number of submissions a journal receives is lower for journals that issue retractions versus journals that issue no retractions. The journals that issued retractions in 2007 show on average a percent decrease in the number of submissions received in 2008.

The last three years (2016-2018) show a reverse trend. We suspect that this is due to the new start journals in the data. There was a mean average of 3.5 years between an article and its retraction, for those retractions in our data set ( $\max =30$ years.) Journals that have been publishing for three years or less are significantly less likely to publish any retractions and therefore are likely to have skewed the data for the last three years.

\section{Journal Reputation: Altmetric Score}

Altmetric score was dropped due to availability of data.

\section{Editorial Processes}

No, or only negligible, difference was found in percentage submission increases when analyzing time to first decision, time to acceptance, and acceptance ratio. These null results are summarized in Table 5.

\section{Case Studies}

Of the 39 responses we received, 4 did not identify a reason for the decline in submissions. In several cases the apparent decline was an artefact of the data caused by a change of submission systems $(n=3)$ or the splitting of the journal into two $(n=3)$. Some respondents identified external factors, such as Impact Factor $(n=4)$ or competitor journals $(n=3)$ as the primary factor. The emergence of "sound science" mega-journals has added to the competition from more traditional specialized journals. Others identified internal journal changes as the primary factor, including a change of editor(s) $(n=2)$, increased rejection rates $(n=3)$, and turnaround times ( $n$ $=2$ ). In several cases the decline in submissions was explained as an intentional editorial strategy to refocus the journal $(n=6)$, or by a move away from commissioned content $(n=4)$. In one case, the decline in submissions was connected by the respondent to a highly publicized case of misconduct by the editor-in-chief. In another case, the decline in submission was explained by imposition of complicated and unnecessary submission requirements (Table 4).

The importance of Impact Factor was a common feature of respondents. One publishing manager said "If authors have to choose between 3 journals with the same scope, they'll go in order of impact factor almost all the time." An editor-in-chief worried about the influence of Impact Factor, noting that some authors chose a competitor journal with a higher Impact Factor, despite claiming "this is a poorly managed and uneven journal, and that papers published there are viewed very differently".

Our evaluation of the factors did not agree with the respondents in every case (Table 4). Impact Factor was raised frequently in the responses, as a secondary factor if not the primary one, but in some cases the decrease 
in Impact Factor or ISI category ranking was relatively small. In two cases the respondents explained the decrease in submissions as an expected fluctuation, which seemed unlikely when given the extremity of the decline. One respondent proposed that the decline in submissions was due the journal rejecting more, when in fact the acceptance rate went up. Retractions was not a factor identified by any of the respondents but, given our data, it seemed to be the most likely factor in at least four cases.

Our statistical analysis did not find a correlation between acceptance rate and submissions, but some of our respondents highlighted a connection. One editor-in-chief, who has worked on several journals, said "if you get a huge spike in rejection rate, you can get a drop in submissions."

Additional desk research looked at social media in the researcher community (e.g. scirev.org; letpub.com; pubpeer.com, Twitter, etc.) regarding the journal and year of each case study. In four cases we identified significant negative comments regarding aspects of peer review at the respective journal, concerning either the process or the outcomes.

We also identified a decline in the industry connected subject discipline of the journals in three of our cases; we suspect that the decline in that industry may correlate with a decline in research output. A respondent to one of our other cases also identified a decline in research funding in the journal's field as a factor.

\section{Limitations}

The analysis of Impact Factor looked at number of submissions, rather than percentage increase, so the average increase is rough and not generalizable across all journals. Future analysis could include repeating these analyses stratified by subject area, or other factors, to improve the accuracy of the coefficient. Also, future analysis could look at relative ISI subject ranking, rather than absolute Impact Factor.

For each year of analysis there is a very small sample of journals with retractions (often under 30 journals), which may cause issues with the accuracy of the results of the t-tests. This analysis does not distinguish between a journal publishing 1 retraction in a single year versus a journal publishing 15. Some journals issued retractions every year, or most years, so no difference can be analyzed.

The Impact Factor is released midway through the calendar year. Retractions might be published at any point in the calendar year. Future analysis could look at submissions by month, perhaps focusing on the 12-month period after the release of the IF or publication of the retraction.

Because the sample includes journals that started publishing within the timeframe (or shortly before), efforts were made to exclude the first and second year of submissions data. However, the submissions growth of a new journal may not be linear and may be uneven.

New journals are unlikely to have an Impact Factor and less likely to have retractions.

\section{Discussion}

There are many factors involved in the number of submissions a journal receives and many of them are beyond the control of the editor or the publisher. The number of research papers being written will be dependent in large part on the amount of research funding available and the number of relevant untapped research areas in that field. (One respondent to our cases noted how the technique covered by the relevant journal is now "commonplace" leading to a decline in new research.) Even where research funding unchanged, submission numbers would still fluctuate, dependent on the incidence of negative or null results (which are commonly not submitted for publication.) Therefore, submission numbers are unlikely to rise evenly. Because there are multiple 
submission options for authors, including both general and specialized journals, there is competition between journals for submissions.

From our data, we identified three factors that have a significant impact on submission numbers. The finding that changes in Impact Factor correlate to changes in submissions is not surprising, as it fits with prior research about authors' selection process. Because Impact Factor is used by various bodies, including universities and funders, to assess published research, authors will inevitably seek to be published in journals with the highest Impact Factor. One of our respondents noted, "it has been unfortunate that some countries and grant agencies set arbitrary limits of what IF journals will or will not count in certain metrics."

ISI ranking is closely linked to Impact Factor, so it is expected that this too would correlate with number of submissions. As ISI \% ranking takes the subject area of the journal into account, this is a useful result that suggests the findings can be generalized, and are not skewed by certain subject areas, which may contain journals with higher average submissions or Impact Factors.

Our third finding is less expected - that retractions correlate with lower submissions. Prior research indicated that journal reputation is an important factor for authors when choosing where to submit. Our case study research found that negative research comments about a journal's peer review occurred in years with significant declines in submissions. Though an isolated case, the decline of submissions following editor-in-chief misconduct seems indicative of a correlation between journal reputation and submissions. To distinguish these reputational effects from Impact Factor, we shall categorize them "peer review reputation" (PRR). (We use "peer review" in the general sense, incorporating the roles of editors and editorial office staff in manuscript evaluation.) We propose that PRR is an important selection criterion for authors and that significant negative PRR will lead to a decrease in submission numbers. We believe this is the reason for the correlation between retractions and submission numbers. (Previous researchers have used retractions as a metric for peer review quality; see (Horbach \& Halffman, 2018).

Whilst in many surveys journal reputation was ranked higher than Impact Factor, it is not possible to use our data to contrast IF and PRR (as measured by retractions) to determine which is the more significant "risk factor" for a decrease in submissions. Our analysis of these two factors involved different tests and different types of analysis, which are not easily comparable. Also, the number of retractions, whilst statistically significant, was relatively small. Changes in Impact Factor correlate to both increases and decreases in submission numbers, whilst retractions only correlate to decreases in submissions. Retractions are also a binary measure; either a retraction was published, or it wasn't. The cases where multiple retractions were published by the same journal in the same year were too few to test for any correlation between number of retractions and number of submissions - we do not expect such a relationship. Furthermore, it may be that trying to rank IF against PRR would be meaningless in any case.

It is worth noting the previous finding that high IF journals published more retractions (Fang \& Casadevall, 2011). Those authors speculate that the correlation may be due to the high IF increasing the incentive for authors to manipulate their results in order to get published, or may be due to higher IF journals coming under more intense scrutiny. Others have proposed that high IF journals favour papers reporting novel and unexpected results, which are disproportionately likely to be retracted later (Agrawal \& Sharma, 2012). Regardless of the cause, this correlation between IF and retractions means that the correlation between these two factors and submissions will not be a simple one; it may be impossible to truly isolate each factor in an analysis.

We found no correlation with submissions for factors such as acceptance rate and turnaround times. One might expect authors to care about the expected speed of dissemination and the likelihood of being published, and there is some indication of this in the literature. We suspect the reason that there was no correlation is that these metrics are not routinely made available to authors. Editors and publishers might consider advertising acceptance rates and turnaround times to assess the impact on submissions. (Pepermans \& Rousseau, 2015) found that, for high-quality papers only, framing the metric as an acceptance rate, rather than a rejection rate, did lead to authors being more likely to submit. 


\section{Conclusion}

We found that IF, ISI ranking, and retractions do have an impact on submissions, but this information needs to be treated with care. There will be natural fluctuations in IF year-on-year. Furthermore, a journal may prioritize strategies for the benefit of their readers that have negatively impact the journal's IF, such as publishing content targeting practitioners or clinicians, which might be highly downloaded but poorly cited.

There are multiple factors that affect submission numbers. We have been able to statistically verify two such factors but there are certainly others, where either the data is not currently available or are more difficult to quantify. Our results do not justify a simplistic focus on only one or two factors.

We do not believe that the correlation between retractions and submission numbers should be considered an incentive for editors or publishers to not publish retractions. Firstly, publishers have a responsibility to maintain the scientific and scholarly literature, which includes the publication of retractions, corrections, and errata. Secondly, whilst retractions correlate with submission numbers, there are many incidences in our data where there was no effect, so it is too simplistic to conclude that retractions cause declines in submissions. Retractions are a quantifiable way of measuring PRR, but certainly not the only aspect of it. Thirdly, as our case studies indicate, negative PPR is transmitted by many means including word-of-mouth (which would be impossible to quantify) and not just via retractions. Lastly, we suspect that not publishing a retraction, where one was justified, would ultimately lead to a greater decline in PPR than publishing the retraction.

We recommend that editors and publishers consider both PRR and IF when analyzing submission numbers. Given that PRR is likely to be a significant factor in determining journal choice, publishers may want to find metrics other than retractions by which to measure it. Journals need sustained or increasing submissions to remain viable, so editors and publishers should aim to maintain a journal's PRR, as well as its IF, to continue to attract submissions. Underinvesting in the practices and processes around peer review may lead to smaller submission numbers and thereby smaller revenues in the longer term.

\section{Acknowledgements}

We gratefully acknowledge the assistance of a number of Wiley colleagues. James Cook did some data collation, Emily Mitic did some data validation, and Tom Broomfield did some data extraction.

Some additional desk research was undertaken by Felicity Ounsworth whilst she was on a work experience placement at Wiley.

\section{Data Availability Statement}

Data about Wiley journals, including submission numbers, turnaround times, and acceptance rates is proprietary.

Retraction data was obtained from Retraction Watch and is open at http://retractiondatabase.org/

\section{Author Contributions}

Thomas Gaston - Conceptualization; Data curation; Investigation; Methodology; Project administration; Supervision; Writing - original draft; Writing - review \& editing;

Francesca Ounsworth - Conceptualization; Data curation; Investigation; Methodology; Writing - review \& editing; 
Emma Jones - Data curation; Investigation; Methodology; Formal analysis; Writing - review \& editing;

Sarah Ritchie - Formal analysis; Methodology; Validation; Writing - review \& editing;

Tessa Senders - Formal analysis; Methodology; Writing - original draft;

\section{Conflict of Interests}

TG, EJ, and SR are all Wiley employees. FO was also a Wiley employee when the research was conducted and has since left the company. TS was an intern at Wiley when the research was analyzed.

Tables

Table 1: Mean increase in number of submissions per every 1 point increase in Impact Factor. Linear regressions show that Impact Factor has a statistically significant impact on the number of submissions a journal receives for each pair of year below. On average, submissions increased by $\sim 100$ manuscripts for each point increase in Impact Factor (actual increases would vary with the size of the journal).

\begin{tabular}{|c|c|c|c|c|c|c|c|c|c|}
\hline & $\begin{array}{l}2010 \# \\
\text { of Subs }\end{array}$ & $\begin{array}{l}2011 \# \text { of } \\
\text { Subs }\end{array}$ & $\begin{array}{c}2012 \# \text { of } \\
\text { Subs }\end{array}$ & $\begin{array}{c}2013 \# \text { of } \\
\text { Subs }\end{array}$ & $\begin{array}{c}2014 \# \text { of } \\
\text { Subs }\end{array}$ & $\begin{array}{c}2015 \# \text { of } \\
\text { Subs }\end{array}$ & $\begin{array}{c}2016 \# \text { of } \\
\text { Subs }\end{array}$ & $\begin{array}{c}2017 \text { \# of } \\
\text { Subs }\end{array}$ & $\begin{array}{c}2018 \# \text { of } \\
\text { Subs }\end{array}$ \\
\hline 2009 IF & 109.72 & 112.37 & & & & & & & \\
\hline 2010 IF & & 100.63 & 97.55 & & & & & & \\
\hline $2011 \mathrm{IF}$ & & & 97.38 & 100.14 & & & & & \\
\hline 2012 IF & & & & 97.01 & 98.65 & & & & \\
\hline 2013 IF & & & & & 102.69 & 99.98 & & & \\
\hline 2014 IF & & & & & & 103.2 & 104.91 & & \\
\hline 2015 IF & & & & & & & 109.56 & 113.5 & \\
\hline 2016 IF & & & & & & & & 110.6 & 115.0 \\
\hline 2017 IF & & & & & & & & & 119.19 \\
\hline
\end{tabular}

Table 2: Mean increase in number of submissions for every $1 \%$ increase in ISI ranking. Linear regressions show that ISI \% ranking is also significantly linked to the number of submissions a journal receives for each pair of years below. On average, submissions increased by $\sim 5$ manuscripts for each $1 \%$ increase in ISI ranking (actual increases would vary with the size of the journal.)

\begin{tabular}{|c|c|c|c|c|c|c|c|c|c|}
\hline & $\begin{array}{l}2010 \\
\text { \# of } \\
\text { Subs }\end{array}$ & $\begin{array}{l}2011 \text { \# of } \\
\text { Subs }\end{array}$ & $\begin{array}{l}2012 \text { \# of } \\
\text { Subs }\end{array}$ & $\begin{array}{l}2013 \text { \# of } \\
\text { Subs }\end{array}$ & $\begin{array}{l}2014 \# \text { of } \\
\text { Subs }\end{array}$ & $\begin{array}{l}2015 \text { \# of } \\
\text { Subs }\end{array}$ & $\begin{array}{l}2016 \# \text { of } \\
\text { Subs }\end{array}$ & $\begin{array}{l}2017 \text { \# of } \\
\text { Subs }\end{array}$ & $\begin{array}{l}2018 \text { \# of } \\
\text { Subs }\end{array}$ \\
\hline 2009 ISI & 4.18 & 4.34 & & & & & & & \\
\hline 2010 ISI & & 4.33 & 4.11 & & & & & & \\
\hline 2011 ISI & & & 4.06 & 4.24 & & & & & \\
\hline 2012 ISI & & & & 4.6 & 4.85 & & & & \\
\hline 2013 ISI & & & & & 4.92 & 4.86 & & & \\
\hline 2014 ISI & & & & & & 4.73 & 4.72 & & \\
\hline 2015 ISI & & & & & & & 4.9 & 5.04 & \\
\hline 2016 ISI & & & & & & & & 5.04 & 5.16 \\
\hline 2017 ISI & & & & & & & & & 5.4 \\
\hline
\end{tabular}

Table 3: Mean increase in number of submissions (retractions vs. no retractions) Note that figures are provided as percentages, so 0.01 would represent a 1\% increase and -0.01 would represent a 1\% decrease, for example.

\begin{tabular}{|c|c|c|c|c|c|c|}
\hline Year & Mean (no retraction) & Mean (retraction) & diff & t-value & df & p-value \\
\hline 2008 & 0.28 & -0.01 & 0.29 & 6.52 & 33.85 & $1.87 \mathrm{e}-07$ \\
\hline 2009 & 0.1 & 0.04 & 0.06 & 2.53 & 30.59 & 0.02 \\
\hline 2010 & 0.1 & 0.03 & 0.07 & 2.56 & 30.13 & 0.02 \\
\hline 2011 & 0.1 & 0.07 & 0.03 & 0.91 & 25.67 & 0.37 \\
\hline
\end{tabular}




\begin{tabular}{|c|c|c|c|c|c|c|}
\hline 2012 & 0.07 & 0.01 & 0.06 & 2.35 & 48.34 & 0.02 \\
\hline 2013 & 0.06 & 0.05 & 0.02 & 0.39 & 53.66 & 0.7 \\
\hline 2014 & 0.06 & 0.02 & 0.04 & 2.05 & 62.1 & 0.04 \\
\hline 2015 & 0.05 & 0.03 & 0.02 & 0.66 & 47.82 & 0.51 \\
\hline 2016 & 0.02 & 0.04 & -0.02 & -0.67 & 58.62 & 0.5 \\
\hline 2017 & 0.03 & 0.08 & -0.04 & -0.97 & 57.35 & 0.34 \\
\hline 2018 & 0.09 & 0.12 & -0.03 & -0.64 & 58.23 & 0.52 \\
\hline
\end{tabular}

Table 4: Primary Reason for Submissions Decline, (a) as identified by respondents, and (b) adjusted following our desk research. Note: we attempted to identify a reason in our research, even where there was no response to our case study request.

\begin{tabular}{|c|c|c|c|}
\hline Category & Respondents & Adjusted following research & diff \\
\hline Bad Peer Review Reputation & & 4 & 4 \\
\hline Change of Editor(s) & 2 & 2 & 0 \\
\hline Competitor journals & 3 & 3 & 0 \\
\hline Decline in Research Output & & 3 & 3 \\
\hline Expected Fluctuation & 2 & & -2 \\
\hline Impact Factor/ISI ranking Decline & 4 & 11 & 7 \\
\hline Intentional Editorial Strategy & 6 & 6 & 0 \\
\hline Journal Split & 3 & 3 & 0 \\
\hline Less commissioned content & 4 & 3 & -1 \\
\hline Moved submission system & 3 & 3 & 0 \\
\hline None Given & 4 & & -4 \\
\hline No Obvious Reason & & 10 & 10 \\
\hline Prior Submissions Spike & 1 & & -1 \\
\hline Rejection Rate & 3 & & -3 \\
\hline Retractions & & 4 & 4 \\
\hline Significant Editor Misconduct & 1 & 1 & 0 \\
\hline Turnaround Times & 2 & 1 & -1 \\
\hline Unnecessary Submission Requirements & 1 & 1 & 0 \\
\hline
\end{tabular}


Table 5: Percentage of journals in sample with submissions increase/decrease for each factor. None of these findings were significant.

\begin{tabular}{|c|c|c|c|c|c|c|c|c|}
\hline & \multicolumn{2}{|l|}{ NPS Score } & \multicolumn{2}{l|}{ Time to First Decision } & \multicolumn{2}{l|}{ Time to Acceptance } & \multicolumn{2}{l|}{ Accept Ratio } \\
\hline & Promoter & Demoter & Increased & Decreased & Increased & Decreased & Increased & Decreased \\
\hline Submissions Increase & 0.62 & 0.7 & 0.65 & 0.68 & 0.68 & 0.67 & 0.68 & 0.67 \\
\hline Submissions Decrease & 0.38 & 0.3 & 0.35 & 0.32 & 0.32 & 0.33 & 0.32 & 0.33 \\
\hline
\end{tabular}

\section{References}

Aarssen, L. W., Tregenza, T., Budden, A. E., Lortie, C. J., Koricheva, J., \& Leimu, R. (2008). Bang for Your Buck: Rejection Rates and Impact Factors in Ecological Journals. The Open Ecology Journal, 1(1), 14-19. https://doi.org/10.2174/1874213000801010014

Adam, D. (2002). The counting house. Nature, 415(6873), 726-729. https://doi.org/10.1038/415726a

Agrawal, A., \& Sharma, A. (2012). Likelihood of False-Positive Results in High-Impact Journals Publishing Groundbreaking Research. Infection and Immunity, 80(3), 1300-1300. https://doi.org/10.1128/iai.06233-11

Calcagno, V., Demoinet, E., Gollner, K., Guidi, L., Ruths, D., \& de Mazancourt, C. (2012). Flows of Research Manuscripts Among Scientific Journals Reveal Hidden Submission Patterns. Science, 338(6110), 1065-1069. https://doi.org/10.1126/science.1227833

Coupe, T. (2004). What Do We Know about Ourselves? on the Economics of Economics. Kyklos, 57(2), 197-215. https://doi.org/10.1111/j.0023-5962.2004.00250.x

Crampsie, T. N. C. (2019). From Journal Selection to Open Access: Practices among Academic Librarian Scholars. Portal: Libraries and the Academy, 19.

Edie, A. H., \& Conklin, J. L. (2019). Avoiding predatory journals: Quick peer review processes too good to be true. Nursing Forum, 54(3), 336-339. https://doi.org/10.1111/nuf.12333

Fang, F. C., \& Casadevall, A. (2011). Retracted Science and the Retraction Index. Infection and Immunity, 79(10), 3855-3859. https://doi.org/10.1128/iai.05661-11

Gibler, K. M., \& Ziobrowski, A. J. (2002). Authors Perceptions and Preferences Among Real Estate Journals. Real Estate Economics, 30(1), 137-157. https://doi.org/10.1111/1540-6229.00033

Heintzelman, M., \& Nocetti, D. (2009). Where Should we Submit our Manuscript? An Analysis of Journal Submission Strategies. The B.E. Journal of Economic Analysis \& Policy, 9(1). https://doi.org/10.2202/1935-1682.2340

Horbach, S. P. J. M., \& Halffman, W. (2018). The ability of different peer review procedures to flag problematic publications. Scientometrics, 118(1), 339-373. https://doi.org/10.1007/s11192-018-2969-2

Nicholas, D., Watkinson, A., Jamali, H. R., Herman, E., Tenopir, C., Volentine, R., ... Levine, K. (2015). Peer review: still king in the digital age. Learned Publishing, 28(1), 15-21. https://doi.org/10.1087/20150104

Pepermans, G., \& Rousseau, S. (2015). The decision to submit to a journal: Another example of a valence-consistent Shift?. Journal of the Association for Information Science and Technology, 67(6), 1372-1383. https://doi.org/10.1002/asi.23491

Phase5Research. (2014). Canadian researchers' publishing attitudes and behaviours.

Salinas, S., \& Munch, S. B. (2015). Where Should I Send It? Optimizing the Submission Decision Process. PLOS ONE, 10(1), e0115451. https://doi.org/10.1371/journal.pone.0115451

Schroter, S., \& Tite, L. (2006). Open Access Publishing and Author-Pays Business Models: A Survey of Authors' Knowledge and Perceptions. Journal of the Royal Society of Medicine, 99(3), 141-148. https://doi.org/10.1177/014107680609900316

Smith, R. (2006). Commentary: The power of the unrelenting impact factor - Is it a force for good or harm?. International Journal of Epidemiology, 35(5), 1129-1130. https://doi.org/10.1093/ije/dyl191

Solomon, D. J., \& Björk, B.-C. (2011). Publication fees in open access publishing: Sources of funding and factors influencing choice of journal. Journal of the American Society for Information Science and Technology, 63(1), 98-107. https://doi.org/10.1002/asi.21660

Swan, A. (1999). 'WHAT AUTHORS WANT': the ALPSP research study on the motivations and concerns of contributors to learned journals. Learned Publishing, 12(3), 170-172. https://doi.org/10.1087/09531519950145742

Swan, A., \& Brown, S. (2004). Authors and open access publishing. Learned Publishing, 17(3), 219-224. https://doi.org/10.1087/095315104323159649

Søreide, K., \& Winter, D. C. (2010). Global survey of factors influencing choice of surgical journal for manuscript submission. Surgery, 147(4), 475-480. https://doi.org/10.1016/j.surg.2009.10.042

Vogel, G. (2011). Open Access Gains Support; Fees and Journal Quality Deter Submissions. Science, 331(6015), $273-273$. https://doi.org/10.1126/science.331.6015.273-a

Willmott, H. (2011). Journal list fetishism and the perversion of scholarship: reactivity and the ABS list. Organization, 18(4), 429442. https://doi.org/10.1177/1350508411403532

Özçakar, L., Franchignoni, F., Kara, M., \& Muñoz, L. S. (2012). Choosing a scholarly journal during manuscript submission: the way how it rings true for physiatrists.. Eur J Phys Rehabil Med, 48, 643-647. 

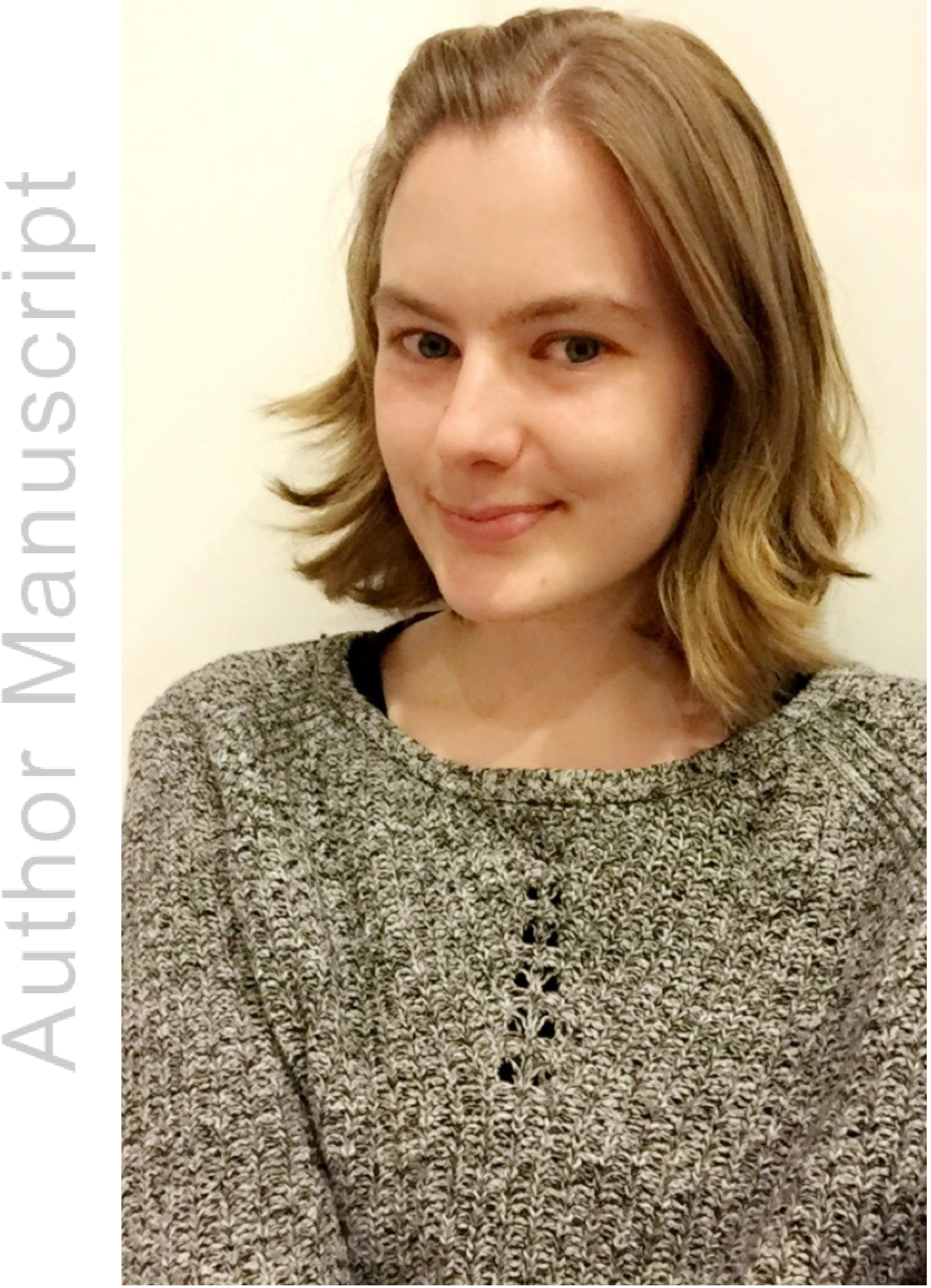

LEAP_1285_emma jones.jpg

This article is protected by copyright. All rights reserved. 


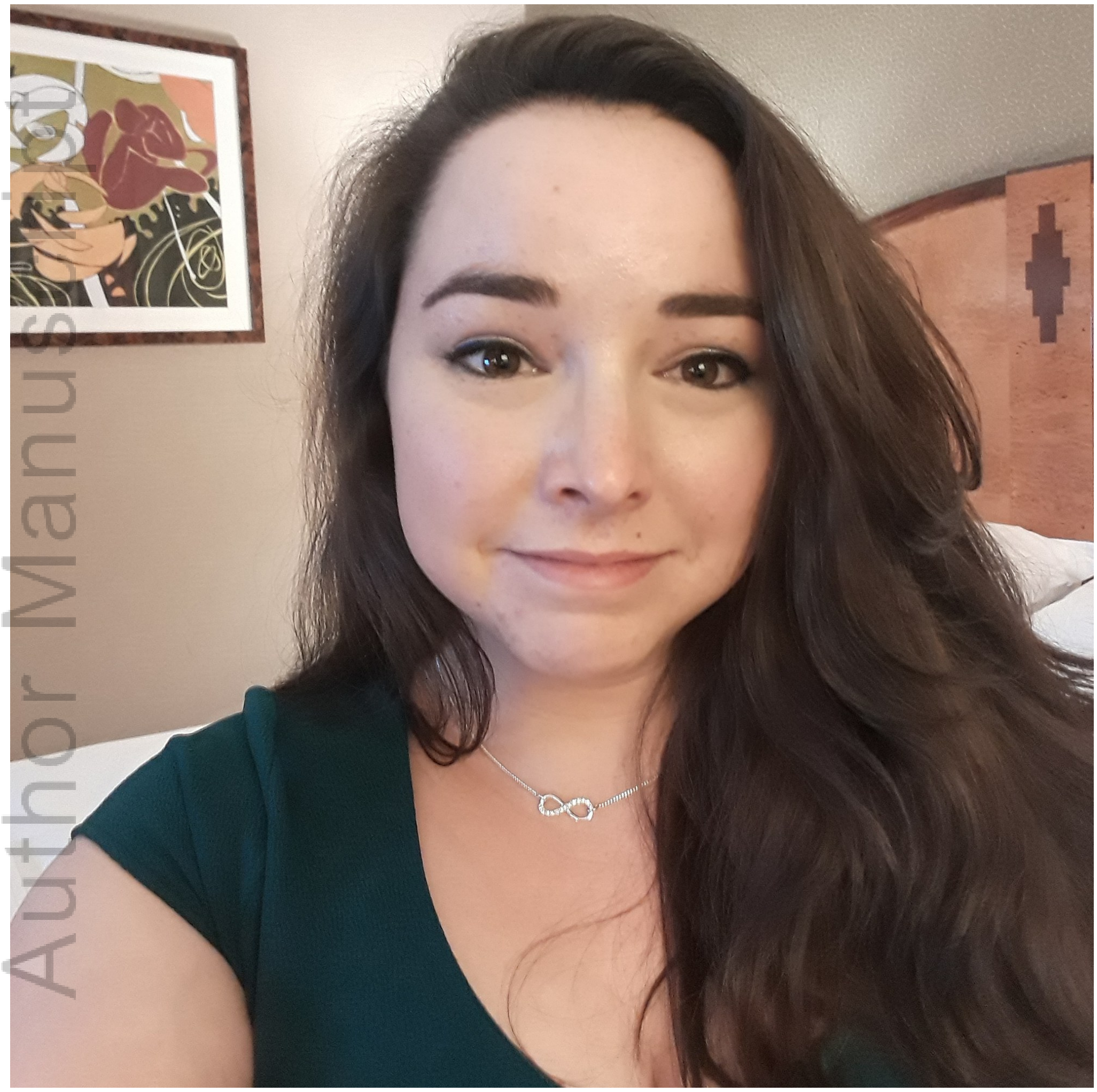

LEAP_1285_fran ounsworth.jpg

This article is protected by copyright. All rights reserved. 


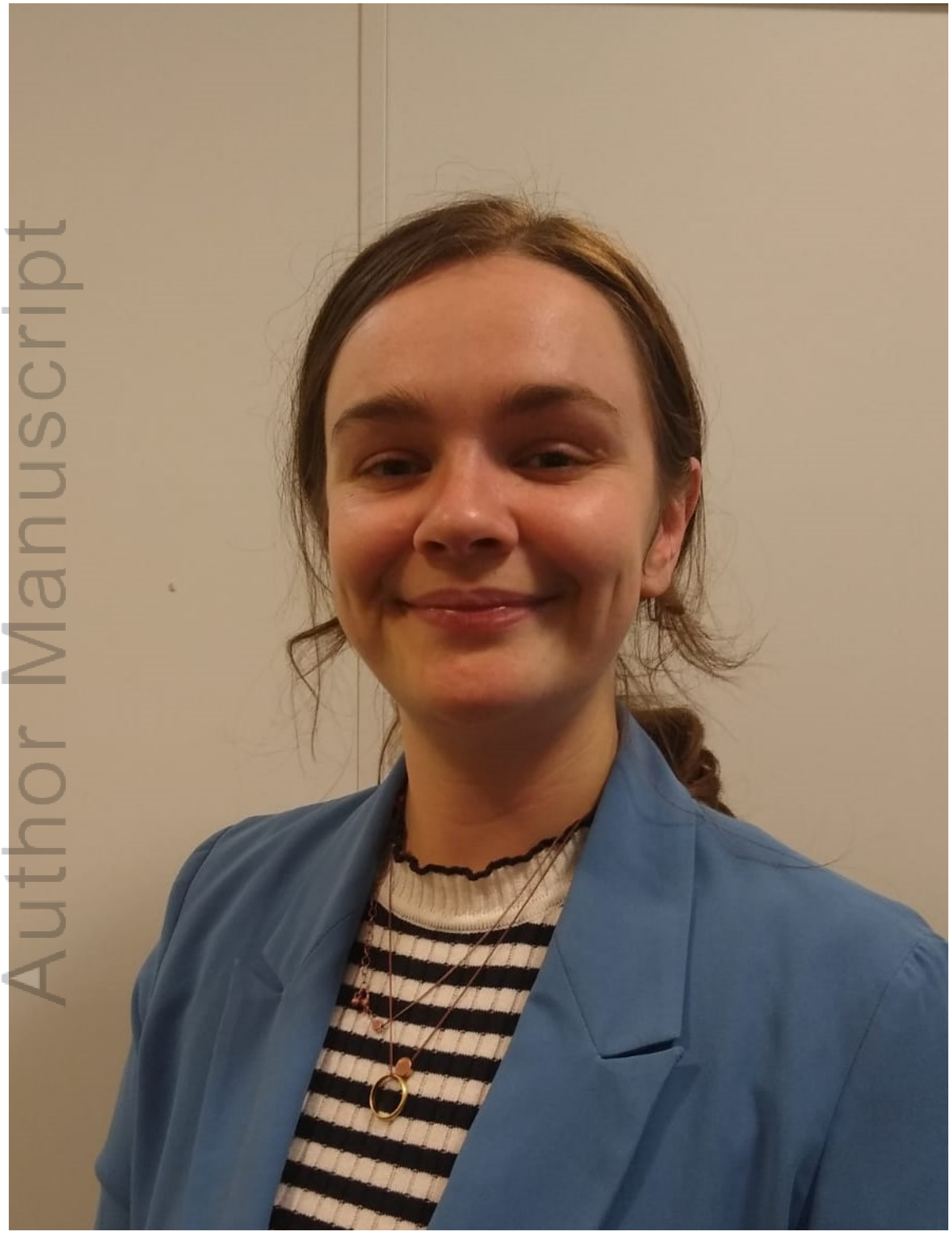

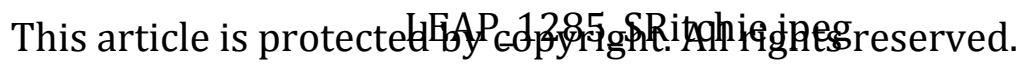




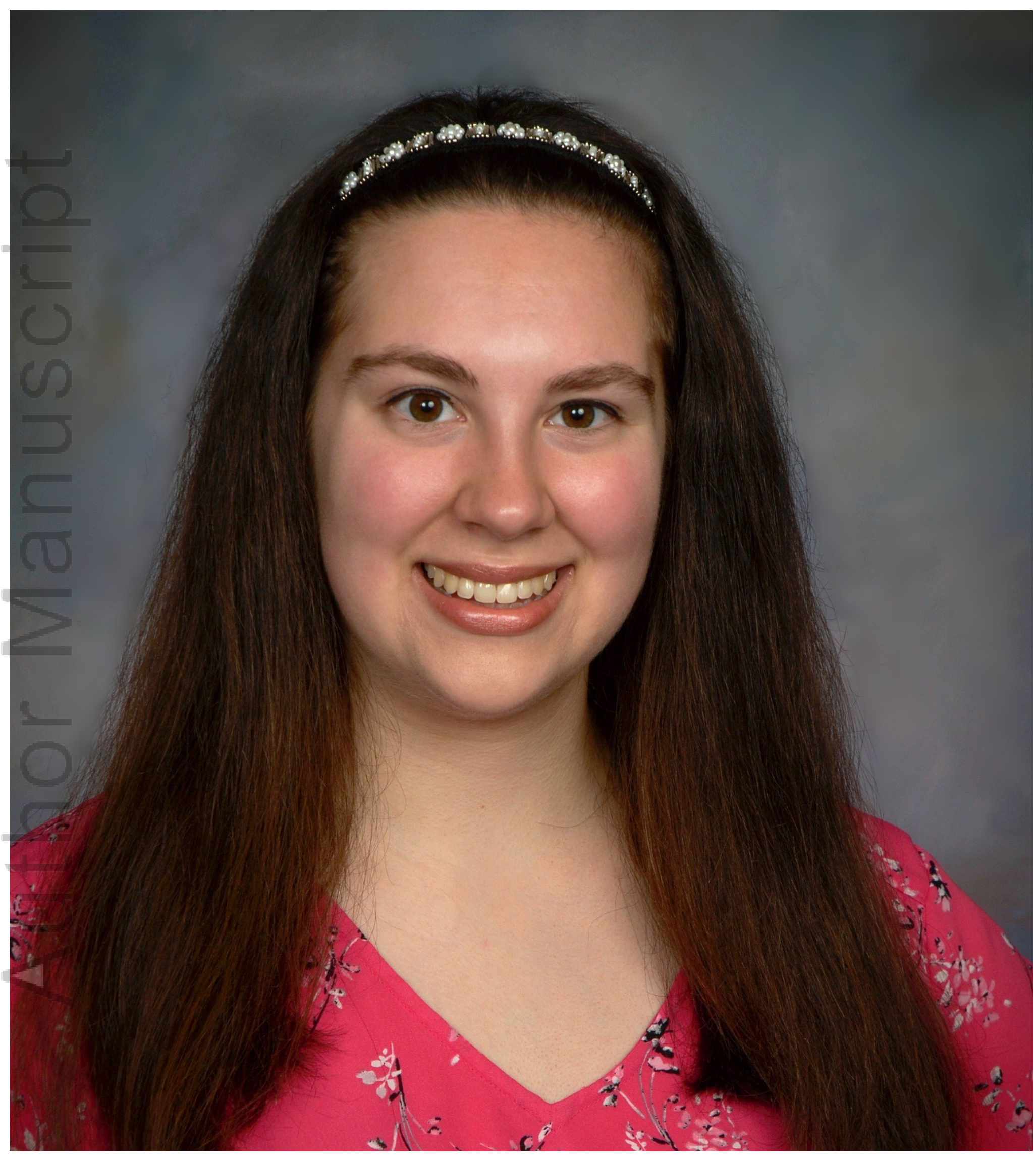

LEAP_1285_tess senders.jpg

This article is protected by copyright. All rights reserved. 


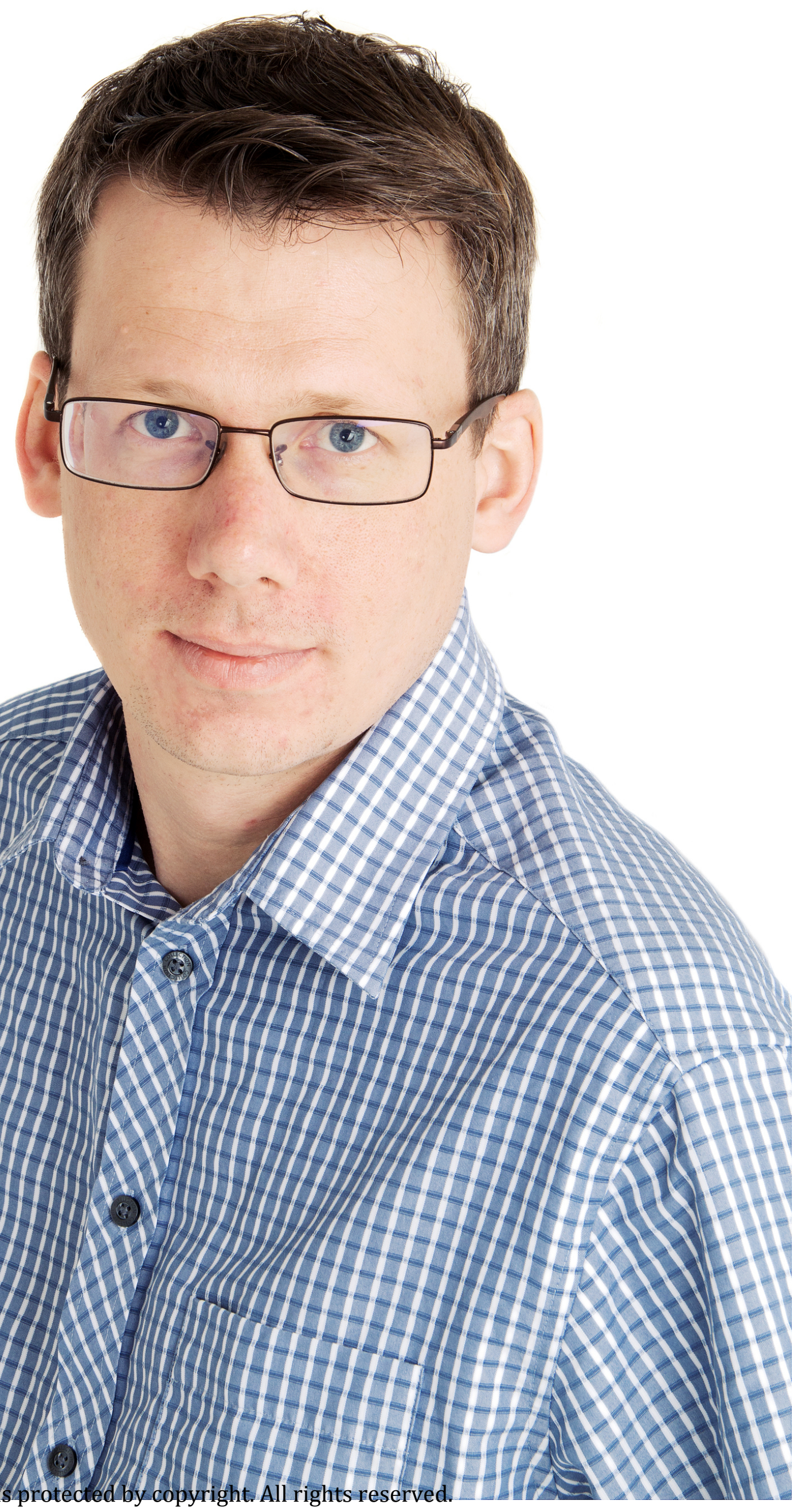

LEAP_1285_tom gaston.jpg 